\title{
Coulisses
}

Revue de théâtre

14 | Printemps 1996

Varia

\section{Cosi Fan Tutte}

$\mathrm{Au}$-delà de l'infime légèreté de l'être

\section{Yvette Marin}

\section{(2) OpenEdition}

\section{Journals}

Édition électronique

URL : http://journals.openedition.org/coulisses/4877

DOI : $10.4000 /$ coulisses.4877

ISSN : 2546-9460

Éditeur

Presses universitaires de Franche-Comté

\section{Édition imprimée}

Date de publication : 1 mai 1996

Pagination : 81-82

ISSN : 1150-594X

\section{Référence électronique}

Yvette Marin, « Cosi Fan Tutte », Coulisses [En ligne], 14 | Printemps 1996, mis en ligne le 20 mars 2019, consulté le 31 octobre 2019. URL : http://journals.openedition.org/coulisses/4877 ; DOI : 10.4000/ coulisses. 4877

Ce document a été généré automatiquement le 31 octobre 2019

Coulisses 


\title{
Cosi Fan Tutte
}

\author{
Au-delà de l'infime légèreté de l'être
}

\section{Yvette Marin}

1 Cosi Fan Tutte fut créé au Burghtheater de Vienne le 26 janvier 1790 et repris quelque deux siècles plus tard à l'Opéra théâtre de Besançon, tout bruissant de la foule des beaux jours qu'amenait le parrainage des Monts Jura. Tout le monde sait bien qu'aujourd'hui le monde de l'entreprise aide le monde de la culture à survivre, lequel le lui rend bien puisqu'il lui assure les feux de la gloire.

\section{En attendant Mozart}

2 Bref, nous étions là, nous les humbles spectateurs, assortis des fanatiques opératiques de tous genres, puis des fanatiques restreints, comme les amoureux de Mozart - ah, Mozart, ah ! - puis des adeptes de l'entreprise cultivée et culturelle. L'opéra craquait de toutes parts en attendant de pouvoir se "ressourcer» au cours de la très prochaine croisière musicale, avec chœurs et orchestre, réservée à la Franche-Comté mélomane. Oui, vous pouvez le dire car ce n'est un secret pour personne, oui, tous de FrancheComté ! On dégustera la cancoillotte sous d'autres cieux, bercés par les vagues! Comme je vous le dis! Et en attendant, on attendait Mozart.

3 Il vint, dans un décor controversé de Thierry Leproust, qui prit un parti minimal : un pan de mur peint d'un feuillage luxuriant qui s'ouvrait, se transformait en boîte, se refermait à loisir. Pour qui était un peu placé sur le côté, comme moi, cela donnait de vilaines choses, comme de voir l'épingle à nourrice qui retient un ourlet.

4 Mais une fois ma critique délivrée, et donc mon cœur soulagé, j'en viens aux compliments. Nous eûmes de jolies voix féminines, des cantatrices crédibles et charmantes, assorties de messieurs presque aussi bien qu'elles, mais pas tout à fait. Ils ont donc peur, ces messieurs, de s'user le coffre? Que donc de timidité dans le souffle, au risque de faire choir le spectateur ! Car il ne faut pas oublier que le spectateur de Cosi, faute d'une meilleure assise, est rivé au chant qui mène l'intrigue et s'y emberlificote. Donc, pas de choix possible pour le spectateur embarqué à l'Opéra 
théâtre : soit il monte, plane et se pâme, soit il ne décolle pas et s'ennuie. Plus tard, dans la croisière, il pourra toujours se jeter à l'eau et aux requins... Mais je glose...

\section{Une musique - ah, vous dis-je !}

5 Le charme opéra - c'est le cas de le dire - et l'opéra fut bon, tout pénétré des résonances harmonieuses particulières, et superbes, que l'on ne retrouve dans aucun autre opéra de Mozart. Ici, le texte de l'opéra buffa de Lorenzo Da Ponte suggère à Mozart déjouer avec les sonorités, puisque les personnages ne sont guère plus que des marionnettes. Une magnifique production donnée jadis au Palais Gantier nous offrait tout Cosi en ombres chinoises. Seules les voix dominaient... Quelle beauté ! Ce sont des souvenirs comme ça, profonds, durables, qui font que la vie vaut la peine d'être vécue... mais qui vous gâchent le présent puisqu'il faut bien comparer, hélas! Alors, pourquoi cette tentation des metteurs en scène du minimal, de la boîte en plaques d'aggloméré fermée par un crochet d'où surgissent les personnages? L'économie qu'on leur impose? Mais plus la période est difficile, plus il faut introduire le rêve et la magie! L'opium du peuple, n'est-ce-pas l'opéra?

6 Revenons à Cosi. On dit que son histoire est fondée sur un fait réel qui défraya la chronique de l'époque: deux officiers avaient entrepris de séduire leurs fiancées respectives, et obtinrent, après efforts louables il faut bien le dire, de forts bons résultats. Cela aurait pu rester trivial, banal, comique ou grotesque, $d u$ « boulevard » du XVIII siècle, une histoire de cocus en somme. Et pourtant, si Mozart rit et se moque, et batifole à son aise comme il aime et comme il sait si bien le faire, il effleure durablement la tragédie, au risque de l'entamer, de s'en faire saigner, et arrache à ses héroïnes des accents désespérés.

7 Rappelons-le, le point de départ de l'intrigue est burlesque, on joue sur les déguisements et les masques pour faire croire que chacun est à sa chacune alors que l'un est avec l'autre. Et si ces demoiselles tombent dans le piège tendu, est-ce bien de leur faute? "Cosi fan tutte»: «Ainsi font-elles toutes», donc il vaut mieux comprendre et pardonner. Pour accéder au tragique de la nature humaine ainsi décrite sans être décriée, Mozart utilise une écriture musicale la plus ornée, dépassant le statut du «bel canto », dont le but principal et avoué est de mettre en valeur une virtuosité vocale. En outre, en dehors des deux grands airs par personnage, comme il se doit, Mozart mêle les voix en duos, trios, quatuors, dont les successions et les alternances épuisent les combinaisons possibles avec un raffinement suprême. Cosi Fan Tutte bénéficie d'une orchestration particulière où les voix dialoguent sans cesse avec des instruments solistes qui s'individualisent pour aller à leur rencontre. Ainsi, les personnages se trouvent décrits, peints et soulignés par les colorations instrumentales. Et là, éclair de génie du metteur en scène, il souligne d'un trait de craie la silhouette des deux femmes, les rendant ainsi interchangeables.

8 On a souvent comparé Cosi à Don Juan et aux Noces de Figaro, sans doute à cause du duo que formait Mozart avec son librettiste Da Ponte dans tous ces opéras. Si on peut, certes, trouver des fils conducteurs communs à ces trois œuvres, il ne me semble pas qu'il faille s'y appesantir. Laissons à Cosi les vertus qui lui sont propres. Dans l'ambiguïté des situations, j'y verrais plutôt l'ombre de Shakespeare dans ses nombreuses comédies. 
9 Ce Cosi plein de fraîcheur et de tendresse est pourtant l'une des dernières œuvres de Mozart puisque celui-ci mourut un an après que la première représentation fut donnée. On connaît l'événement, l'enterrement dans la fosse commune, les rares amis. On sait aussi que lorsque Mozart composa Cosi, il était déjà criblé de dettes, malade, découragé. Dans cet opéra si plein de grâces, il a déjà revêtu les couleurs du poète et accédé à la sérénité. C'est peut- être là que se situe le «message » de Cosi, au-delà des vicissitudes du quotidien et de l'infime légèreté de l'être.

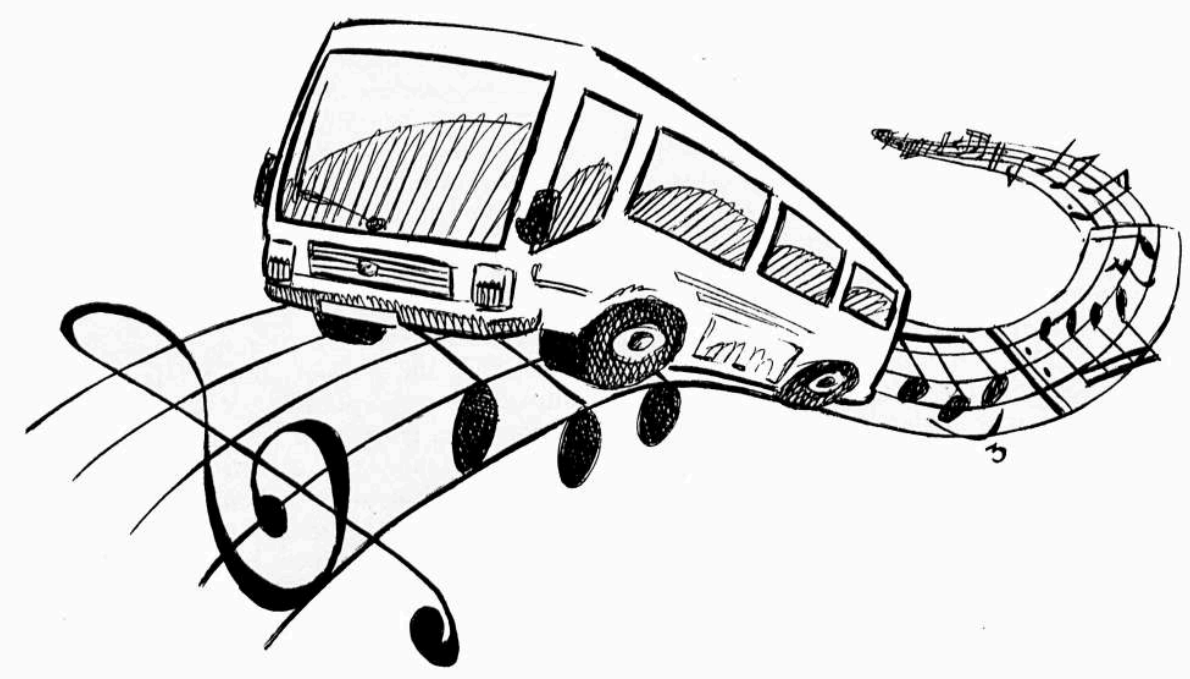

Dessin Raphaël Baud 